\title{
Efficacy and Safety of Direct-Acting Oral Anticoagulants (DOACs) in the Overweight and Obese
}

\author{
Kimberley Doucette $\mathbb{D}^{1,2}$, Hira Latif, ${ }^{1}$ Anusha Vakiti ${ }^{1 D},{ }^{1,3}$ Eshetu Tefera $\left(D,{ }^{4}\right.$ \\ Bhavisha Patel $(\mathbb{D})^{1,5}$ and Kelly Fitzpatrick $(\mathbb{1})^{1}$ \\ ${ }^{1}$ Medstar Washington Hospital Center, Departments of Internal Medicine and Hematology and Oncology, Washington, DC, USA \\ ${ }^{2}$ Medstar Georgetown University Hospital, Departments of Hematology and Oncology, Washington, DC, USA \\ ${ }^{3}$ Medical College of Georgia, Department of Hematology and Oncology, Augusta, GA, USA \\ ${ }^{4}$ Medstar Health Research Institute, Biostatistics and Biomedical Informatics, Hyattsville, MD, USA \\ ${ }^{5}$ National Heart, Lung and Blood Institute, Hematology Branch, Bethesda, MD, USA
}

Correspondence should be addressed to Kimberley Doucette; kimberley.doucette@medstar.net

Received 20 February 2020; Accepted 25 April 2020; Published 23 May 2020

Academic Editor: Debra A. Hoppensteadt; dhoppen@luc.edu

Copyright ( 92020 Kimberley Doucette et al. This is an open access article distributed under the Creative Commons Attribution License, which permits unrestricted use, distribution, and reproduction in any medium, provided the original work is properly cited.

\begin{abstract}
Obesity plays an essential role in the safety of pharmacologic drugs. There is paucity of data for direct oral anticoagulants (DOACs) in the obese, despite these agents becoming more widely used. The primary and secondary objectives of this study were to assess the safety and efficacy of DOACs in the overweight and obese populations when used for primary prophylaxis in the setting of non-valvular atrial fibrillation (NVAF) and for treatment of venous thromboembolisms (VTE). We conducted a retrospective cohort study in a large tertiary care center and obtained data through review of electronic health records. Among patients with NVAF and VTE on apixaban, there were no differences in rates of major bleeding (MB) and clinically relevant nonmajor bleeding (CRNMB) in the overweight and obese populations when compared to normal weight and underweight individuals. The multivariate adjusted analysis for rivaroxaban found that the odds of CRNMB for patients with BMI $<25$ was 5.37 (95\% CI 1.50-19.32) times higher than that of BMI $\geq 25$. Moreover, patients on medications that had known interactions with DOACs had 6.40 times higher odds of CRNMB than patients without such interactions (95\% CI 1.49-27.57), which was not accounted for by the effects of aspirin and plavix alone. Efficacy was similar between all weight groups, for both apixaban and rivaroxaban. These results support previous analyses preformed in the large phase III trials and confirm that apixaban and rivaroxaban are safe in the overweight and obese.
\end{abstract}

\section{Introduction}

Obesity is a major healthcare challenge that is estimated to escalate to a global epidemic in the following years [1]. Therefore, it is essential to assess the healthcare needs of this population in terms of pharmacologic modifications required for this subset to provide effective treatment and prevent adverse events. Obesity is a well-established risk factor for developing VTE (venous thromboembolism), and vitamin $\mathrm{K}$ antagonists (VKA) have historically been the mainstay of anticoagulation treatment [2-5] given lack of data in this specific population. Direct oral anticoagulants (DOAC) are now approved for use as primary prophylaxis in patients with nonvalvular atrial fibrillation to prevent stroke and systemic embolism, as well as treatment for deep vein thrombosis (DVT) and pulmonary embolism (PE). They are also deemed safe for use as prophylaxis for venous thromboembolism (VTE) in patients undergoing knee or hip replacement surgery. These newer agents have shown equal efficacy as low-molecular weight heparin (LMWH) and VKAs $[6,7]$. In addition, studies have shown no differences in efficacy to VKAs or LMWH in overweight and obese patients [8-13]. Additionally, safety parameters, namely, major bleeding and clinically relevant nonmajor bleeding (CRNMB), are reported to be lower in all patients taking DOACs when compared to VKAs [14]. Their fixed 
dosing, limited dietary interactions, and reduced need for routine monitoring make them a convenient alternative to the traditional VKA.

Subgroup analyses in various randomized clinical trials have evaluated the efficacy and safety measures in obese patients and showed no differences in efficacy compared to conventional VKAs or enoxaparin. Fourteen percent of the patients in the EINSTEIN trial and 20\% in the AMPLIFY trial were over $100 \mathrm{~kg}$, and the results for efficacy and safety outcomes were consistent and did not suggest a need for dose modification for weight. However, there have been no dedicated large prospective randomized control trials to address the use of DOAC in the obese. Despite limited data, these agents are being used in clinical practice in the real world population [8-13, 15-17].

Pharmacokinetics and pharmacodynamics studies in this domain have been conflicting and not conclusive. In a healthy volunteer study, an inverse relationship between the drug and patient weight was observed $[9,18]$. Drug exposures were found to be decreased along with reduced concentrations and shortened half-lives (time to steady state) in patients with $\mathrm{BMI}>40 \mathrm{~kg} / \mathrm{m}^{2}$. Other smaller studies and case reports have demonstrated stable pharmacokinetics and pharmacodynamics in obese patients for both rivaroxaban and apixaban $[7,19,20]$. To date, there exists no strong evidence of clinical efficacy and safety in obese patients on therapeutic DOACs. As such, the general recommendations are to avoid DOACs in the extremes of weight (e.g., $<50 \mathrm{~kg},>120 \mathrm{~kg}$, or $\mathrm{BMI} \geq 35 \mathrm{~kg} / \mathrm{m}^{2}$ ) unless VKA is contraindicated [21-23]. In addition, there are some concerns about their interaction with other cytochrome p-450 3A4 modifying medications such as amiodarone, verapamil, antifungal agents such as ketoconazole, and antibiotics such as erythromycin in obese patients [24].

Given the lack of robust clinical evidence of using these newer agents in the obese population, we aim to evaluate the efficacy and safety of DOACs (apixaban, rivaroxaban, and dabigatran) in overweight and obese patients with NVAF and VTE. Our study was performed in the diverse population of patients being treated at the largest hospital in the District of Columbia.

\section{Methods}

We conducted a retrospective cohort study of the inpatient and outpatient population at Medstar Washington Hospital Center in Washington DC, recruited between January 2014 and December 2015. Patients were included if prescribed DOACs (either rivaroxaban, apixaban, or dabigatran) for VTE, NVAF, or stroke prophylaxis and were 18 years or older. Exclusion criteria were inconsistent follow-up or documented noncompliance after initiation of therapy. We collected baseline demographic data including age, race, gender, BMI, length of follow-up time, and comorbidities. We also assessed concomitant use of CYP3A inhibitors and inducers (nondihydropyridine calcium channel blockers, amiodarone, antiarrhythmics, protease inhibitors, and antiepileptic (carbamazepine/phenytoin)) and antiplatelets (aspirin and/or clopidogrel).
Our primary endpoint was safety events up to 2 years after initiation of DOAC for NVAF patients and 6 months for VTE patients in overweight and obese patients (BMI $<25$ versus $\geq 25$ and $<30$ versus $\geq 30$, respectively). Safety measures were defined as any bleeding defined by the International Society on Thrombosis and Haemostasis (ISTH) criteria. Major bleeding was defined as overt bleeding with a drop in hemoglobin of at least $2 \mathrm{~g} / \mathrm{dL}$ or requiring 2 units of packed red blood cells at a critical site. Clinically relevant nonmajor bleeding (CRNMB) was defined as clinically overt bleeding leading to hospitalization, medical/surgical intervention, or change in anticoagulation treatment but did not satisfy criteria of major bleeding [25]. Combined bleeding included both CRNMB and MB. Our secondary outcome was efficacy and included strokes for the NVAF cohort and recurrent VTE in the VTE cohort with follow-up up to 2 years and 6 months, respectively, after initiation of a DOAC. Patients were stratified by BMI $\left(\mathrm{kg} / \mathrm{m}^{2}\right)$ to assess the efficacy and safety in the obese patient population. BMI categories include normal range (18-24.99), overweight (25-29.99), obese class I (30-34.99), obese class II (35-39.99), and obese class III (>40.00) per WHO criteria.

Summary statistics were performed on baseline characteristics in the various study groups using means, standard deviations, and proportions (if categorical). A two sample $t$ test was used to examine differences in the averages of continuous variables between groups (NVAF vs VTE). Chisquare and Fisher's exact tests were used as appropriate to investigate differences for categorical variables.

To assess the relations between outcomes and BMI, our statistical analyses included chi-square, Fisher's exact (when cells had fewer than 5 counts), and logistic regression. If patients had missing variables, they were removed from analyses. We also performed analysis on combined BMI groups, $<25$ versus $\geq 25$ and $<30$ versus $\geq 30$. Our logistic regression models adjusted for the comorbidities, dose of DOAC, and use of CYP3A inhibitors and inducers or antiplatelet agents. A $p$ value $<0.05$ was considered statistically significant. We used the SAS statistical software.

\section{Results}

3.1. Patient Characteristics. We collected data for a total of 398 patients from January 2014 to December 2015 who received DOACs for NVAF or VTE at Medstar Washington Hospital Center (MWHC) in either an inpatient or outpatient encounter. The patient demographics are described in Table 1 . In the NVAF cohort, males were slightly overrepresented (57\%) and median age was 72 years. Seventynine percent of the patients had BMI $>25$ and $47 \%$ had BMI $>30$, with $13 \%$ having a BMI $>40$.

In contrast, the VTE cohort had slight predominance of females (58\%) with a median age of 65 years. African Americans were majority, with a similarly large portion of patients having BMI $>25$ (75\%), and $26 \%$ with BMI $>30$, and $12 \%$ with a BMI $>40$. The two groups differed with respects to history of hypertension, heart failure, and presence of interacting medications and use of aspirin or clopidogrel; the 
TABLE 1: Baseline characteristics of study patients.

\begin{tabular}{|c|c|c|c|}
\hline Characteristics & $\operatorname{AF}(N=289)$ & $\operatorname{VTE}(N=109)$ & $p$ value \\
\hline Age (yr) & $72 \pm 12$ & $65 \pm 17$ & $<0.0001$ \\
\hline Male, no. (\%) & $165(57)$ & $46(42)$ & 0.0079 \\
\hline Race, no. (\%) & & & $<0.0001$ \\
\hline AA & $135(47)$ & $78(72)$ & \\
\hline White & $139(48)$ & $27(25)$ & \\
\hline Asian & $2(1)$ & $1(1)$ & \\
\hline Hispanic & $3(1)$ & 0 & \\
\hline BMI, no. $(\%)^{*}$ & & & 0.8337 \\
\hline Underweight $(<18.5)$ & $2(1)$ & $1(0.9)$ & \\
\hline Normal (18.5-24.9) & $60(21)$ & $24(22)$ & \\
\hline Overweight (25.0-29.9) & $90(31)$ & $26(24)$ & \\
\hline Obesity (class I) (30.0-34.9) & $63(22)$ & $28(26)$ & \\
\hline Very obese (class II) (35.0-39.9) & $38(13)$ & $15(14)$ & \\
\hline Extreme obesity (class III) $(>40)$ & $36(13)$ & $13(12)$ & \\
\hline \multicolumn{4}{|l|}{ DOAC, no. $(\%)$} \\
\hline Apixaban & $126(44)$ & $18(17)$ & 0.2263 \\
\hline $2.5 \mathrm{mg}$ twice daily & $15(12)$ & $4(22)$ & \\
\hline $5 \mathrm{mg}$ twice daily & $111(88)$ & $14(78)$ & \\
\hline Rivaroxaban & $127(44)$ & $87(79)$ & 0.4532 \\
\hline $10 \mathrm{mg}$ once daily & $2(2)$ & $0(0)$ & \\
\hline $15 \mathrm{mg}$ once daily & $16(13)$ & $13(15)$ & \\
\hline $20 \mathrm{mg}$ once daily & $109(86)$ & $74(85)$ & \\
\hline Dabigatran & $36(13)$ & $3(3)$ & 0.0828 \\
\hline $75 \mathrm{mg}$ twice daily & $2(6)$ & $1(33)$ & \\
\hline $150 \mathrm{mg}$ twice daily & $34(94)$ & $2(67)$ & \\
\hline History of hypertension, no. (\%) & $251(87)$ & $79(73)$ & 0.0007 \\
\hline History of diabetes mellitus, no. (\%) & $75(26)$ & $27(25)$ & 0.0090 \\
\hline History of heart failure, no. (\%) & $98(34)$ & $16(15)$ & 0.0002 \\
\hline History of HIV, no. (\%) & $4(1)$ & $5(4.6)$ & 0.0553 \\
\hline History of malignancy, no. (\%) & $41(14)$ & $13(12)$ & 0.5571 \\
\hline History of lung disease, no. (\%) & $41(14)$ & $19(17)$ & 0.4199 \\
\hline History of treatment failure, no. (\%) & $12(4)$ & $10(9)$ & 0.0506 \\
\hline Presence of any interacting medication, no. (\%) & $164(57)$ & $36(33)$ & $<0.0001$ \\
\hline Presence of either aspirin and/or plavix, no. (\%) & $116(40)$ & $27(25)$ & 0.0044 \\
\hline History of prior bleed(s) before AC, no. (\%) & $21(7)$ & $11(10)$ & 0.3553 \\
\hline
\end{tabular}

${ }^{*}$ Categories as described per WHO criteria.

NVAF group had a larger proportions of all these factors. Rivaroxaban was prescribed more commonly in the VTE group (79\%) compared to the NVAF group (44\%). Only a small fraction of patients were on dabigatran in both groups.

3.2. NVAF Primary Outcome: Safety. When BMI $<30$ was compared to $\mathrm{BMI} \geq 30$, in the apixaban and rivaroxaban groups, rates of bleeding (major, CRNMB, and combined bleeds) were not statistically different. In contrast, CRNMB and combined bleeds (CRNMB and $\mathrm{MB}$ ) in the rivaroxaban group were more frequent in patients with normal BMI $(<25)$ compared to overweight BMI $(>25) \quad(p=0.0004)$ (Table 2). A logistic regression model was then conducted adjusting for age, sex, race, prior stroke, presence of interacting medications, dose of DOAC, and history of prior bleeds. This multivariate analysis showed that when adjusting for these covariates, the odds of CRNMB for patients with BMI <25 was 5.37 (95\% CI 1.50-19.32) times higher than that of patients with $\mathrm{BMI} \geq 25$ (Table 3 ). It also showed that patients with drug interactions had 6.4 times higher odds of CRNMB than patients without the presence
TABLE 2: Safety outcome for NVAF in all patients.

\begin{tabular}{|c|c|c|c|c|}
\hline \multirow{2}{*}{$\begin{array}{l}\text { Safety } \\
\text { outcome }\end{array}$} & \multicolumn{2}{|c|}{ Rivaroxaban $(N=127)$} & \multicolumn{2}{|c|}{ Apixaban $(N=126)$} \\
\hline & Event rate $(\%)$ & $p$ value & Event rate $(\%)$ & $p$ value \\
\hline \multicolumn{5}{|c|}{ Major bleeding } \\
\hline $\mathrm{BMI}<25$ & $0 / 25=0$ & 1.000 & $4 / 32=12.5$ & 0.036 \\
\hline $\mathrm{BMI} \geq 25$ & $2 / 102=2.0$ & & $0 / 94=0$ & \\
\hline $\mathrm{BMI}<30$ & $0 / 68=0$ & 0.214 & $4 / 71=5.6$ & 0.131 \\
\hline $\mathrm{BMI} \geq 30$ & $2 / 59=3.4$ & & $0 / 55=0$ & \\
\hline \multicolumn{5}{|c|}{ Clinically relevant nonmajor bleeding (CRNMB) } \\
\hline BMI $<25$ & $11 / 25=44.0$ & 0.004 & $2 / 32=6.3$ & 0.352 \\
\hline $\mathrm{BMI} \geq 25$ & $15 / 102=14.7$ & & $13 / 94=13.8$ & \\
\hline $\mathrm{BMI}<30$ & $15 / 68=22.1$ & 0.634 & $9 / 71=12.7$ & 1.000 \\
\hline $\mathrm{BMI} \geq 30$ & $11 / 59=18.6$ & & $6 / 55=10.9$ & \\
\hline \multicolumn{5}{|c|}{ Combined bleeding* } \\
\hline BMI $<25$ & $11 / 25=44.0$ & 0.006 & $6 / 32=18.8$ & 0.570 \\
\hline $\mathrm{BMI} \geq 25$ & $17 / 102=16.7$ & & $13 / 94=24.4$ & \\
\hline $\mathrm{BMI}<30$ & $15 / 68=22.1$ & 0.997 & $13 / 71=18.3$ & 0.250 \\
\hline $\mathrm{BMI} \geq 30$ & $13 / 59=22.0$ & & $6 / 55=10.9$ & \\
\hline
\end{tabular}

${ }^{*}$ Combined bleeding is a composite outcome of major bleeding and clinically relevant nonmajor bleeding. Chi-square, and Fisher's exact tests for safety outcomes (major bleeding, CRNMB, and combined bleeding) in patients with $\mathrm{BMI}<25$ versus $\geq 25$ in the NVAF cohort. 
TABLE 3: Multivariate analysis of rivaroxaban and CRNMB in overweight patients.

\begin{tabular}{lcc}
\hline Variables & Odds ratio estimate & 95\% Wald confidence interval \\
\hline BMI $\geq 25$ versus $<25$ & 5.37 & $1.50-19.31$ \\
Age & 1.02 & $0.98-1.07$ \\
Male & 0.74 & $0.23-2.41$ \\
Caucasian versus African American & 0.54 & $0.17-1.74$ \\
Dose of rivaroxaban & 0.96 & $0.80-1.15$ \\
Interacting medications & 6.40 & $1.49-27.57$ \\
Aspirin & 0.53 & $0.14-2.04$ \\
Plavix & 16.68 & $0.44-630.68$ \\
History of prior bleeding & 0.20 & $0.03-1.18$ \\
\hline
\end{tabular}

This table presents the multivariate logistical regression model for rivaroxaban and odds ratios for outcome of CRNMB in overweight patients compared to normal weight controls in the NVAF cohort.

of drug interactions (95\% CI 1.49-27.57). Neither aspirin nor clopidogrel alone accounted for this effect. The same multivariate analysis for patients with $\mathrm{BMI}<30$ versus $\geq 30$ showed no statistically significant difference in rates of CRNMB (Table 4). For apixaban, comparing BMI $<25$ to $\mathrm{BMI} \geq 25$, both CRNMB and MB rates were found to be similar between the groups. The sample size for dabigatran was too small, and therefore, no statistical analyses could be performed on these patients.

3.3. VTE Primary Outcome: Safety. For rivaroxaban in the VTE cohort, bleeding rates were similar across all BMIs. There was no significant relationship across the different BMI groups for major bleeding, CRNMB, and combined bleeding (Table 5). The sample sizes for these groups were smaller than the NVAF cohort. The sample size for dabigatran and apixaban were too small, and therefore no statistical analysis could be performed on these patients.

3.4. Secondary Outcomes: Efficacy. For both apixaban and rivaroxaban in the NVAF cohort, stroke rates were similar across all BMIs; however, TIA events were uncommon in the rivaroxaban group, making an analysis impossible. Fisher's exact test revealed no significant relationship across the different BMI groups and stroke/ TIA rates (Table 6). For the VTE cohort, recurrence rate of VTE at 6 months appeared similar across all BMIs for both apixaban and rivaroxaban. Sample size was again very small for the apixaban group (19 patients in total). Fisher's exact test for recurrence rate in the apixaban group and chi-square for recurrence rate in the rivaroxaban group revealed no significant relationship across the different BMI groups (Table 6).

\section{Discussion}

Due to paucity of data regarding use of DOACs in the obese population, we have to base our clinical decisions about their use on available evidence. Our study provides further evidence of comparable efficacy and safety of apixaban and rivaroxaban in the obese patients with AF and VTE. Our results suggest that there is an increased rate of clinically relevant nonmajor bleeding in patients on rivaroxaban with normal or lower weights (BMI <25) as opposed to overweight and obese patients, independent of dose of DOAC. This finding was not seen in the apixaban group. We found no increased bleeding risk in obese patients with $\mathrm{BMI} \geq 30$ compared to patients with $\mathrm{BMI}<30$. Our sample size was too small to assess the safety profile of patients with a BMI over 40; however, we did include a high proportion of overweight and obese patients. Our limitations are that it is a retrospective study with potential for selection bias and has a small sample size. We were unable to collect enough data for dabigatran in the VTE and AF cohorts or for apixaban in the VTE cohort. To fully assess efficacy, a longer follow-up period will be required. Our safety results support the current literature and represent a generalizable tertiary center population. Unfortunately, a lot of bleeding episodes occurred in outpatient setting, and we were unable to collect data on coagulation studies. Another limitation is the lack of documentation on herbal supplements taken over the counter, which may have interactions with DOACs. We collected data on use of over the counter aspirin, which is often documented in progress notes, though is not linked with pharmacy records, making documentation variable. A recent large retrospective study, looking at safety measures and efficacy in the extreme obesity weight class (morbidly obese) on DOACs, found no increased bleeding risk and similar efficacy compared to patients on warfarin [26]. There was another retrospective study in 2016, that showed no significant difference between rates of bleeding when comparing patients that were underweight $(<60 \mathrm{~kg})$ versus those with a weight in the normal range versus the overweight (>120 kg) (major bleeding and all bleeding); however, there was a trend towards significance. There were increased rates of bleeding in both underweight and overweight patients compared to normal weight. Important limitations of this study include a small sample size [27]. Our results show the contrary with an increased bleeding risk in lower weight individuals taking rivaroxaban.

Clinical data on DOAC use in the obese and morbidly obese population is limited. Large phase III trials had analyses of varying weight cutoffs, which make direct comparisons across the heterogeneous groups difficult. Guidelines published in 2016 reported DOACs to be generally safe in obese patients with bleeding rates that are comparable to VKAs. Similar evidence from the AMPLIFY, 
TABLE 4: Multivariate analysis of rivaroxaban and CRNMB in obese patients.

\begin{tabular}{lcc}
\hline Variables & Odds ratio estimate & 95\% Wald confidence interval \\
\hline BMI $\geq 30$ versus $<30$ & 1.15 & $0.34-3.89$ \\
Age & 1.052 & $1.00-1.11$ \\
Male & 0.59 & $0.19-1.84$ \\
Caucasian versus African American & 0.67 & $0.22-2.03$ \\
Dose of rivaroxaban & 0.98 & $0.82-1.17$ \\
Interacting medications & 6.73 & $1.70-26.74$ \\
Aspirin & 0.63 & $0.18-2.21$ \\
Plavix & 9.21 & $0.25-341.92$ \\
History of prior bleeding & 0.27 & $0.05-1.48$ \\
\hline
\end{tabular}

This table presents the multivariate logistical regression model for rivaroxaban and odds ratios for outcome of CRNMB in obese patients compared to normal weight controls in the NVAF cohort.

TABLE 5: Safety outcome for VTE in all patients.

\begin{tabular}{|c|c|c|}
\hline \multirow{2}{*}{ Safety outcome } & \multicolumn{2}{|c|}{ Rivaroxaban $(N=86)$} \\
\hline & Event rate $(\%)$ & $p$ value \\
\hline \multicolumn{3}{|l|}{ Major bleeding } \\
\hline BMI $<25$ & $1 / 16=6.3$ & 1.000 \\
\hline $\mathrm{BMI} \geq 25$ & $4 / 69=5.8$ & \\
\hline $\mathrm{BMI}<30$ & $3 / 38=7.9$ & 0.653 \\
\hline $\mathrm{BMI} \geq 30$ & $2 / 47=4.3$ & \\
\hline \multicolumn{3}{|c|}{ Clinically relevant nonmajor bleeding (CRNMB) } \\
\hline BMI $<25$ & $2 / 16=12.5$ & 1.000 \\
\hline $\mathrm{BMI} \geq 25$ & $8 / 69=11.6$ & \\
\hline $\mathrm{BMI}<30$ & $4 / 38=10.5$ & 1.000 \\
\hline $\mathrm{BMI} \geq 30$ & $6 / 47=12.8$ & \\
\hline \multicolumn{3}{|c|}{ Combined bleeding* } \\
\hline BMI $<25$ & $3 / 16=18.8$ & 1.000 \\
\hline $\mathrm{BMI} \geq 25$ & $12 / 69=17.4$ & \\
\hline $\mathrm{BMI}<30$ & $7 / 38=18.4$ & 0.866 \\
\hline $\mathrm{BMI} \geq 30$ & $8 / 48=16.7$ & \\
\hline
\end{tabular}

${ }^{*}$ Combined bleeding is a composite outcome of major bleeding and clinically relevant nonmajor bleeding. Chi-square and Fisher's exact tests for safety outcomes (major bleeding, CRNMB, and combined bleeding) in patients with BMI $<25$ versus $\geq 25$ in the VTE cohort.

TABLE 6: Secondary outcome: efficacy and relationship with BMI in all patients.

\begin{tabular}{|c|c|c|c|c|}
\hline \multirow{2}{*}{ Efficacy outcome } & \multicolumn{2}{|c|}{ Rivaroxaban } & \multicolumn{2}{|c|}{ Apixaban } \\
\hline & Event rate $(\%)$ & $p$ value & Event rate $(\%)$ & $p$ value \\
\hline \multicolumn{5}{|c|}{ Stroke in the AF cohort } \\
\hline BMI $<25$ & $1 / 25=4.0$ & 1.000 & $0 / 31=0$ & 0.574 \\
\hline $\mathrm{BMI} \geq 25$ & $4 / 102=3.9$ & & $3 / 94=3.2$ & \\
\hline $\mathrm{BMI}<30$ & $3 / 68=4.4$ & 1.000 & $1 / 70=1.4$ & 0.582 \\
\hline $\mathrm{BMI} \geq 30$ & $2 / 59=3.4$ & & $2 / 55=3.6$ & \\
\hline \multicolumn{5}{|c|}{ TIA in the AF group } \\
\hline BMI 25 & $0 / 25=0$ & N/A & $0 / 31=0$ & 1.000 \\
\hline $\mathrm{BMI} \geq 25$ & $0 / 102=0$ & & $2 / 94=2.1$ & \\
\hline $\mathrm{BMI}<30$ & $0 / 68=0$ & N/A & $2 / 70=2.9$ & 0.503 \\
\hline $\mathrm{BMI} \geq 30$ & $0 / 59=0$ & & $0 / 55=0$ & \\
\hline \multicolumn{5}{|c|}{ Recurrence in the VTE cohort } \\
\hline BMI $<25$ & $2 / 16=12.5$ & 0.315 & $1 / 8=12.5$ & 1.000 \\
\hline $\mathrm{BMI} \geq 25$ & $4 / 69=5.8$ & & $1 / 11=9.1$ & \\
\hline $\mathrm{BMI}<30$ & $3 / 38=7.9$ & 1.000 & $2 / 11=18.2$ & 0.485 \\
\hline $\mathrm{BMI} \geq 30$ & $3 / 47=6.4$ & & $0 / 8=0$ & \\
\hline
\end{tabular}

Chi-square and Fisher's exact tests for efficacy outcomes (major bleeding, CRNMB and combined bleeding) in patients with BMI $<25$ versus $\geq 25$ in both VTE and $\mathrm{AF}$ cohorts. 
Hokusai-VTE, EINSTEIN-DVT, EINSTEIN-PE, and ROCKET-AF trials also support the safety of DOACs in patients with a higher BMI [8-12]. The exception to this trend was seen in the AMPLIFY trial, where subgroup analysis showed significantly improved bleeding rates among the obese when comparing apixaban to VKA $[8,23]$. Our data supports the safety of DOAC use in patients with higher BMIs by demonstrating no increased bleeding event rates in the apixaban group and lower bleeding event rates in the rivaroxaban group in patients with a BMI over 30 .

In addition, our results show that the presence of interacting medications (concomitant use of CYP3A inhibitors and inducers along with antiplatelet agents) is also an independent risk factor for bleeding in patients with BMIs under 25 and under 30. Although our sample size was too small to decipher which medication group was more likely to lead to increased bleeding, we were able to assess that aspirin and plavix alone did not account for these findings. A recent systemic review found no statistical difference for clinical outcomes including major bleeding or intracranial bleeding in patients taking amiodarone and DOAC concomitantly [28]. Both ARISTOTLE and ROCKET-AF post hoc analyses have shown no impact of interacting drugs on bleeding risk although strong CYP3A inhibitors and inducers were excluded. However, ROCKET$\mathrm{AF}$ found increased risk of major bleeding with concomitant dual antiplatelet use in the RE-LY trial and for aspirin in the ROCKET-AF trial [29-31]. Our results suggest that patients taking medication with potential drug interactions should be monitored closely for bleeding.

Importantly, there was no difference in efficacy across different BMIs supporting the findings of larger clinical trials; however, again, sample size in relation to follow-up duration was small, making interpretation difficult. Ideally, a longer follow-up time would allow us to capture more events and more appropriately assess efficacy.

In summary, our results support previous findings that DOACS are safe in the obese; however, the retrospective nature of our study and small sample size remain important limitations. Future efforts to design larger prospective randomized trials inclusive of more patients with BMIs over 25-40 would be required to validate that the safety and efficacy of DOACs are similar across all BMIs.

\section{Data Availability}

The datasets used and/or analysed during the current study are available from the corresponding author upon reasonable request.

\section{Ethical Approval}

This retrospective cohort study was reviewed by IRB \#3 Washington in accordance with expedited review procedures on 11 March 2017.

\section{Conflicts of Interest}

The authors have no conflicts of interest.

\section{Authors' Contributions}

Kimberley Doucette was a major contributor in collecting and interpreting the data, creating tables, and writing the manuscript. Hira Latif, Anusha Vakiti, and Bhavisha Patel helped in collecting and interpreting the data, creating tables, and writing the manuscript. Kelly Fitzpatrick created the idea and format for the study and contributed to the writing of the manuscript and tables. Eshetu Tefera analysed the patient data. All authors read and approved the final manuscript.

\section{References}

[1] T. Kelly, W. Yang, C.-S. Chen, K. Reynolds, and J. He, "Global burden of obesity in 2005 and projections to 2030," International Journal of Obesity, vol. 32, no. 9, pp. 1431-1437, 2008.

[2] M. T. Severinsen, S. R. Kristensen, S. P. Johnsen, C. Dethlefsen, A. Tjønneland, and K. Overvad, "Anthropometry, body fat, and venous thromboembolism," Circulation, vol. 120, no. 19, pp. 1850-1857, 2009.

[3] M. Abdollahi, M. Cushman, and F. Rosendaal, "Obesity: risk of venous thrombosis and the interaction with coagulation factor levels and oral contraceptive use," Thrombosis and Haemostasis, vol. 89, no. 3, pp. 493-498, 2003.

[4] K. H. Borch, S. K. Braekkan, E. B. Mathiesen et al., "Abdominal obesity is essential for the risk of venous thromboembolism in the metabolic syndrome: the Tromsø study," Journal of Thrombosis and Haemostasis, vol. 7, no. 5, pp. 739-745, 2009.

[5] P. L. Lutsey, B. A. Virnig, S. B. Durham et al., "Correlates and consequences of venous thromboembolism: the Iowa Women's Health Study," American Journal of Public Health, vol. 100, no. 8, pp. 1506-1513, 2010.

[6] T. van der Hulle, J. Kooiman, P. L. den Exter, O. M. Dekkers, F. A. Klok, and M. V. Huisman, "Effectiveness and safety of novel oral anticoagulants as compared with vitamin $\mathrm{K}$ antagonists in the treatment of acute symptomatic venous thromboembolism: a systematic review and meta-analysis," Journal of Thrombosis and Haemostasis, vol. 12, no. 3, pp. 320-328, 2014.

[7] J. Domienik-Karlowicz and P. Pruszczyk, "The use of anticoagulants in morbidly obese patients," Journal of Cardiology, vol. 23, pp. 12-16, 2016.

[8] G. Agnelli, H. R. Buller, A. Cohen et al., "Oral apixaban for the treatment of acute venous thromboembolism," New England Journal of Medicine, vol. 369, no. 9, pp. 799-808, 2013.

[9] S. J. Connolly, M. D. Ezekowitz, S. Yusuf et al., "Dabigatran versus warfarin in patients with atrial fibrillation," New England Journal of Medicine, vol. 361, no. 12, pp. 1139-1151, 2009.

[10] V. T. E. I. Hokusai, H. R. Buller, H. Decousus et al., "Edoxaban versus warfarin for the treatment of symptomatic venous thromboembolism," New England Journal of Medicine, vol. 369, pp. 1406-1415, 2013.

[11] E. Investigators, R. Bauersachs, S. D. Berkowitz et al., "Oral rivaroxaban for symptomatic venous thromboembolism," New England Journal of Medicine, vol. 363, pp. 2499-2510, 2010.

[12] C. B. Granger, J. H. Alexander, J. J. V. McMurray et al., "Apixaban versus warfarin in patients with atrial fibrillation," New England Journal of Medicine, vol. 365, no. 11, pp. 981992, 2011. 
[13] M. R. Patel, K. W. Mahaffey, J. Garg et al., "Rivaroxaban versus warfarin in nonvalvular atrial fibrillation," New England Journal of Medicine, vol. 365, no. 10, pp. 883-891, 2011.

[14] R. C. P. Makam, D. C. Hoaglin, D. D. McManus et al., "Efficacy and safety of direct oral anticoagulants approved for cardiovascular indications: systematic review and metaanalysis," PLoS One, vol. 13, no. 5, Article ID e0197583, 2018.

[15] R. P. Giugliano, C. T. Ruff, E. Braunwald et al., "Edoxaban versus warfarin in patients with atrial fibrillation," New England Journal of Medicine, vol. 369, no. 22, pp. 2093-2104, 2013.

[16] E.-P. Investigators, H. R. Buller, M. H. Prins et al., "Oral rivaroxaban for the treatment of symptomatic pulmonary embolism," New England Journal of Medicine, vol. 366, pp. 1287-1297, 2012.

[17] S. Schulman, A. K. Kakkar, S. Z. Goldhaber et al., "Treatment of acute venous thromboembolism with dabigatran or warfarin and pooled analysis," Circulation, vol. 129, no. 7, pp. 764-772, 2014.

[18] V. V. Upreti, J. Wang, Y. C. Barrett et al., "Effect of extremes of body weight on the pharmacokinetics, pharmacodynamics, safety and tolerability of apixaban in healthy subjects," British Journal of Clinical Pharmacology, vol. 76, no. 6, pp. 908-916, 2013.

[19] R. Pathak, P. Karmacharya, S. Giri et al., "Meta-analysis on efficacy and safety of new oral anticoagulants for venous thromboembolism prophylaxis in overweight and obese postarthroplasty patients," Blood Coagulation \& Fibrinolysis, vol. 26, no. 6, pp. 635-642, 2015.

[20] D. Kubitza, M. Becka, M. Zuehlsdorf, and W. Mueck, "Body weight has limited influence on the safety, tolerability, pharmacokinetics, or pharmacodynamics of rivaroxaban (BAY 59-7939) in healthy subjects," The Journal of Clinical Pharmacology, vol. 47, no. 2, pp. 218-226, 2007.

[21] A. E. Burnett, C. E. Mahan, S. R. Vazquez, L. B. Oertel, D. A. Garcia, and J. Ansell, "Guidance for the practical management of the direct oral anticoagulants (DOACs) in VTE treatment," Journal of Thrombosis and Thrombolysis, vol. 41, no. 1, pp. 206-232, 2016.

[22] K. K. E. K. Graves and S. A. Johnson, "Use of oral anticoagulants in obese patients," JSM Atherosclerosis, vol. 2, p. 1035, 2017.

[23] K. Martin, J. Beyer-Westendorf, B. L. Davidson, M. V. Huisman, P. M. Sandset, and S. Moll, "Use of the direct oral anticoagulants in obese patients: guidance from the SSC of the ISTH," Journal of Thrombosis and Haemostasis, vol. 14, no. 6, pp. 1308-1313, 2016.

[24] H. L. Forbes and T. M. Polasek, "Potential drug-drug interactions with direct oral anticoagulants in elderly hospitalized patients," Therapeutic Advances in Drug Safety, vol. 8, no. 10, pp. 319-328, 2017.

[25] S. Kaatz, D. Ahmad, A. C. Spyropoulos, and S. Schulman, "Definition of clinically relevant non-major bleeding in studies of anticoagulants in atrial fibrillation and venous thromboembolic disease in non-surgical patients: communication from the SSC of the ISTH," Journal of Thrombosis and Haemostasis, vol. 13, no. 11, pp. 2119-2126, 2015.

[26] M. Kushnir, Y. Choi, R. Eisenberg et al., "Efficacy and safety of direct oral factor xa inhibitors in 795 morbidly obese patients," in Proceedings of the ASH Annual Meeting, p. 332, San Diego, CA, USA, December 2018.

[27] L. Talamo, H. S. Maitland, S. Palkimas, and D. White, "Safety of doacs in patients of extreme weight," Blood, vol. 128, no. 22, p. 1450, 2016.
[28] F. Lupercio, J. Romero, B. Peltzer et al., "Efficacy and safety outcomes of direct oral anticoagulants and amiodarone in patients with atrial fibrillation," The American Journal of Medicine, vol. 131, no. 5, pp. e1-e573, 2018.

[29] T. Hellwig and M. Gulseth, "Pharmacokinetic and pharmacodynamic drug interactions with new oral anticoagulants," Annals of Pharmacotherapy, vol. 47, no. 11, pp. 1478-1487, 2013.

[30] R. Shah, A. Hellkamp, Y. Lokhnygina et al., "Use of concomitant aspirin in patients with atrial fibrillation: findings from the ROCKET AF trial," American Heart Journal, vol. 179, pp. 77-86, 2016.

[31] J. Jaspers Focks, M. A. Brouwer, D. M. Wojdyla et al., "Polypharmacy and effects of apixaban versus warfarin in patients with atrial fibrillation: post hoc analysis of the ARISTOTLE trial," BMJ, vol. 353, p. i2868, 2016. 